Article

\title{
Proposal of a Computational Approach for Simulating Thermal Bosonic Fields in Phase Space
}

\author{
Alessandro Sergi ${ }^{1,2}, * \mathbb{C}$, Roberto Grimaudo ${ }^{3}$, Gabriel Hanna ${ }^{4}$ and Antonino Messina ${ }^{2,5}$ \\ 1 Dipartimento di Scienze Matematiche e Informatiche, Scienze Fisiche e Scienze della Terra, Università degli \\ Studi di Messina, viale F. Stagno d'Alcontres 31, 98166 Messina, Italy \\ 2 Istituto Nazionale di Fisica Nucleare, Sez. di Catania, 95123 Catania, Italy; antonino.messina@unipa.it \\ 3 Dipartimento di Fisica e Chimica, Università degli Studi di Palermo, Via Archirafi 36, I-90123 Palermo, Italy; \\ roberto.grimaudo01@unipa.it \\ 4 Department of Chemistry, University of Alberta, 11227 Saskatchewan Drive Edmonton, Edmonton, \\ AB T6G 2G2, Canada; gabriel.hanna@ualberta.ca \\ 5 Dipartimento di Matematica e Informatica, Università degli Studi di Palermo, Via Archirafi 34, \\ I-90123 Palermo, Italy \\ * Correspondence: asergi@unime.it; Tel.: +39-090-676-5485
}

Received: 29 October 2019; Accepted: 25 November 2019; Published: 3 November 2019

check for updates

\begin{abstract}
When a quantum field is in contact with a thermal bath, the vacuum state of the field may be generalized to a thermal vacuum state, which takes into account the thermal noise. In thermo field dynamics, this is realized by doubling the dimensionality of the Fock space of the system. Interestingly, the representation of thermal noise by means of an augmented space is also found in a distinctly different approach based on the Wigner transform of both the field operators and density matrix, which we pursue here. Specifically, the thermal noise is introduced by augmenting the classical-like Wigner phase space by means of Nosé-Hoover chain thermostats, which can be readily simulated on a computer. In this paper, we illustrate how this may be achieved and discuss how non-equilibrium quantum thermal distributions of the field modes can be numerically simulated.
\end{abstract}

Keywords: Wigner function; bosonic field; temperature control; molecular dynamics simulation

\section{Introduction}

According to quantum field theory, the vacuum is filled by fluctuating quantum fields. Such fields are present both in condensed matter systems [1-6] and, more generally, in empty space-time, where their existence is believed to be linked to dark matter and dark energy [7-16]. Universal phenomena such as the emergence of order, symmetry breaking, and phase transitions are related to the thermal degrees of freedom the first time in order to stress that we are not using the expression in a literal meaning. of the fields $[17,18]$. Hence, the study of the thermal excitation of the quantum vacuum is of interest to many research areas [19-23]. In thermo field dynamics [24-26], the vacuum state is generalized to a thermal vacuum state by doubling the dimension of the Fock space of the original vacuum. The additional dimension of the Fock space represent the degrees of freedom of the thermal bath, which are involved in the excitation and de-excitation processes of the thermal system.

In this paper, we illustrate an approach to simulate the thermal distributions of bosonic fields [27-34] on a computer, which exploits the Wigner formulation of quantum field theory [35-40]. When considering a free field, it is useful to first express the field operators and density matrices in terms of creation and annihilation operators. The creation and annihilation operators are then mapped onto canonical position and momentum operators, which are finally Wigner transformed. 
When the initial state of the field is pure, the Wigner distribution function of the field is expressed as an infinite product of the Wigner distribution functions [41-43] of the single field modes. This is also the case for a quantum thermal state of an infinite set of free field modes, i.e., the thermal Wigner function of the field is an infinite product of the thermal Wigner functions of the modes. In practice, it is possible to simulate quantum thermal distributions with a different temperature for each mode and to define a time-dependent temperature. This is achieved by means of a technique called massive Nosé-Hoover chain (NHC) thermostatting [44,45], which, in our case, involves coupling a separate NHC thermostat $[44,45]$ to each mode. Since one requires a method that can be practically implemented on a computer, the Fock space is truncated to become a many-dimensional (but finite) Hilbert space.

It should be noted that, in spite of their theoretical differences, the Wigner representation of the thermal vacuum that is obtained in our approach is analogous to the one given in thermo field dynamics [24-26]. As in thermo field dynamics, where the additional degrees of freedom represent the thermal bath, the Wigner phase space of the thermal system is augmented by the NHC degrees of freedom [44,45]. In practice, because each NHC can often be realized with just two Nosé-Hoover thermostats chained to each other, the dimension of the thermal Wigner phase space is tripled.

A Wigner representation of the modes of the field [46] has certain technical and conceptual advantages. First, since each mode is independently thermostatted, it is easy to generate a non-equilibrium quantum thermal distribution of the field by assigning each mode a different thermodynamic temperature. Secondly, there is no technical difficulty in making the mode temperatures time dependent. As will be explained below, this feature can be exploited to simulate the passage from a quantum thermal distribution of the modes to a classical one. The generalization of our formalism to situations where the field is coupled to a spin system [47-57] is left for future work. Such an endeavour could lead to new computational experiments on spin dynamics, which to the best of our knowledge, would be novel. We propose one such computational experiment in the Conclusions.

The paper is organized as follows. In Section 2, we show how to represent the dynamics and averages of bosonic field modes in Wigner phase space. In Section 3, we discuss how a bosonic thermal state is described within the Wigner representation of quantum field theory. We also illustrate how to control the temperature of each field mode on a computer. Finally, our conclusions and perspectives are given in Section 4 .

\section{Wigner Formulation of Bosonic Field Theory}

Let us consider a quantum bosonic free field, confined to a finite region of space. This field possesses discrete mode frequencies, $\omega_{J}$, and bosonic creation and annihilation operators, $\hat{a}_{J}$ and $\hat{a}_{J}^{\dagger}$, where $J$ is an integer. The Hamiltonian operator of the free field reads [38,46]:

$$
\hat{H}_{\left(\hat{a}, \hat{a}^{\dagger}\right)}=\sum_{J} \hbar \omega_{J}\left(\hat{a}_{J}^{\dagger} \hat{a}_{J}+\frac{1}{2}\right)
$$

Let us now define the following canonical transformation:

$$
\begin{aligned}
& \hat{a}_{J}=\frac{1}{\sqrt{2 \hbar}}\left(\lambda_{J} \hat{Q}_{J}+\frac{i \hat{P}_{J}}{\lambda_{J}}\right), \\
& \hat{a}_{J}^{\dagger}=\frac{1}{\sqrt{2 \hbar}}\left(\lambda_{J} \hat{Q}_{J}-\frac{i \hat{P}_{J}}{\lambda_{J}}\right),
\end{aligned}
$$

where $\hat{Q}_{J}$ and $\hat{P}_{J}$ are Hermitian operators obeying the canonical commutation relation $\left[\hat{Q}_{J}, \hat{P}_{J^{\prime}}\right]=i \hbar \delta_{J J^{\prime}}$. The scaling factor $\lambda_{J}$ is equal to $\sqrt{m_{J} \omega_{J}}$ when the field consists of oscillators of mass $m_{J}$ or is equal to 
$\lambda_{J}=\sqrt{\hbar}\left(\omega_{J} / c\right)$ in the case of an electromagnetic field [46]. Upon substituting Equations (2) and (3) in Equation (1), we obtain another representation for the discretized quantum free field Hamiltonian,

$$
\hat{H}_{(\hat{Q}, \hat{P})}=\sum_{J}\left[\frac{\omega_{J} \hat{P}_{J}^{2}}{2 \lambda_{J}^{2}}+\frac{\omega_{J} \lambda_{J}^{2} \hat{Q}_{J}^{2}}{2}\right]
$$

Since any initial state of the field can be expanded in the basis of Fock states, we consider a multimode Fock state. This can be written as:

$$
\begin{aligned}
|\Phi\rangle & =\left|n_{1}, n_{2}, \ldots, n_{K}, \ldots\right\rangle=\frac{\left(\hat{a}_{1}^{\dagger}\right)^{n_{1}}}{\sqrt{n_{1}+1}} \frac{\left(\hat{a}_{2}^{\dagger}\right)^{n_{2}}}{\sqrt{n_{2}+1}} \cdots \frac{\left(\hat{a}_{K}^{\dagger}\right)^{n_{K}}}{\sqrt{n_{K}+1}} \cdots|0\rangle \\
& =\prod_{J=1}^{\infty} \frac{\left(\hat{a}_{J}^{\dagger}\right)^{n_{J}}}{\sqrt{n_{J}+1}}|0\rangle \equiv \prod_{J=1}^{\infty}\left|\Phi_{J}\right\rangle,
\end{aligned}
$$

where $n_{J}$ is the occupation number of state $J$. To each state $\left|\Phi_{J}\right\rangle$, one can associate a single mode density matrix, i.e., $\hat{\rho}_{J}=\left|\Phi_{J}\right\rangle\left\langle\Phi_{J}\right|$. Therefore, the total density matrix of the field is an infinite tensor product of the single mode density matrices:

$$
\hat{\rho}=\prod_{J=1}^{\infty} \hat{\rho}_{J}
$$

We can now define the Wigner transform [41-43] of each mode's density matrix as:

$$
W_{J}\left(Q_{J}, P_{J}\right)=\frac{1}{(2 \pi \hbar)^{d}} \int d Z e^{i P \cdot Z / \hbar}\left\langle Q_{J}-\frac{Z}{2}\left|\hat{\rho}_{J}\right| Q_{J}+\frac{Z}{2}\right\rangle,
$$

where $d$ is the dimension of the spatial region in which the field is confined. The Wigner distribution function of the field is therefore given by:

$$
W(Q, P)=\prod_{J=1}^{\infty} W_{J}\left(Q_{J}, P_{J}\right),
$$

where the field-mode coordinates, $(Q, P)=\left(Q_{1}, Q_{2}, \ldots, P_{1}, P_{2}, \ldots\right)$, are c-numbers in Wigner phase space $[42,43]$.

We now consider the quantum averages of generic bosonic field operators, $\mathcal{O}$, in the second quantized form:

$$
\langle\hat{\mathcal{O}}(t)\rangle=\operatorname{Tr}(\hat{\rho}(t) \hat{\mathcal{O}}) .
$$

If $\mathcal{O}$ is symmetrically ordered with respect to the $\hat{a}_{J}$ and $\hat{a}_{J}^{\dagger}$ operators [46], that is,

$$
\hat{\mathcal{O}}=\prod_{J=1}^{\infty} \sum_{m, n=0}^{\infty} b_{n m} \frac{1}{2}\left[\left(\hat{a}_{J}^{\dagger}\right)^{n} \hat{a}_{J}^{m}+\hat{a}_{J}^{m}\left(\hat{a}_{J}^{\dagger}\right)^{n}\right] \equiv \prod_{J=1}^{\infty} \hat{\mathcal{O}}_{J},
$$

where $b_{n m}$ are expansion coefficients, then we can introduce the Wigner transform of $\mathcal{O}$ as:

$$
\mathcal{O}_{W}(Q, P)=\prod_{J=1}^{\infty} \int d Z_{J} e^{i P_{J} \cdot Z_{J} / \hbar}\left\langle Q_{J}-\frac{Z_{J}}{2}\left|\hat{\mathcal{O}}_{J}\right| Q_{J}+\frac{Z_{J}}{2}\right\rangle .
$$

Under the restriction for the field observables mentioned above, averages can then be calculated as [46]:

$$
\langle\hat{\mathcal{O}}(t)\rangle=\operatorname{Tr}(\hat{\rho}(t) \hat{\mathcal{O}})=\int d X W(Q, P, t) \mathcal{O}_{W}(Q, P),
$$


where, in practice, in order to obtain the Wigner transform of operators $\mathcal{O}$ obeying the condition mentioned above, one first uses the canonical transformations in Equations (2) and (3) and then replaces the operators $\left(\hat{Q}_{J}, \hat{P}_{J}\right)$ with the c-numbers $\left(Q_{J}, P_{J}\right)$. For example, the Wigner transform of the field Hamiltonian in Equation (1) reads:

$$
H_{(Q, P)}=\sum_{J}\left[\frac{P_{J}^{2}}{2 \mu_{J}}+\frac{\mu_{J} \omega_{J}^{2} Q_{J}^{2}}{2}\right]=\sum_{J} H_{W}^{J}\left(Q_{J}, P_{J}\right)
$$

where $\mu_{J}=\lambda_{J}^{2} / \omega_{J}$. Equation (13) is just the c-number version of Equation (4).

In the Wigner representation, the quantum vacuum field equations in the Heisenberg picture:

$$
\begin{aligned}
\frac{d}{d t} \hat{a}_{J}(t) & =\frac{i}{\hbar}\left[\hat{H}_{\left(\hat{a}, \hat{a}^{\dagger}\right)}, \hat{a}_{J}(t)\right], \\
\frac{d}{d t} \hat{a}_{J}^{\dagger}(t) & =\frac{i}{\hbar}\left[\hat{H}_{\left(\hat{a}, \hat{a}^{\dagger}\right)}, \hat{a}_{J}^{\dagger}(t)\right],
\end{aligned}
$$

become:

$$
\frac{d}{d t} X_{J}=-H_{X} \overleftarrow{\nabla} \mathcal{B} \vec{\nabla} X_{J}(t)
$$

where $X=\left(X_{1}, X_{2}, \ldots, X_{J}, \ldots\right)$ (with $\left.X_{J}=\left(Q_{J}, P_{J}\right)\right), \nabla=\partial / \partial X$, and the direction of the arrow indicates the direction in which the operator acts. The matrix $\mathcal{B}$ is the constant symplectic matrix [58]:

$$
\mathcal{B}=\left[\begin{array}{cc}
0 & \mathbf{1} \\
-1 & 0
\end{array}\right],
$$

whose specific form gives rise to the Poisson bracket [58] on the right hand side of Equation (16) (where 1, for example, denotes an infinite-dimensional block diagonal matrix of ones). Equation (16) is equivalent to Equations (14) and (15), showing that the quantum dynamics of a harmonic system may be exactly mapped onto a classical-like time evolution. In this case, the quantum character of the system enters the description through the initial conditions.

In the next sections, we will see how the use of the Wigner representation allows one to exploit the many numerical algorithms originating from molecular dynamics simulation in order to propagate the degrees of freedom represented in phase space.

\section{Computer Simulation of Thermal Field States}

The field Hamiltonian in Equation (1) is isomorphic to a Hamiltonian of a collection of non-interacting harmonic oscillators [59-62]. The thermal Wigner function, $W_{J}^{T}$, of a harmonic mode has the following analytical form [46]:

$$
W_{J}^{T}\left(Q_{J}, P_{J}\right)=\frac{\omega_{J} \tilde{\beta}_{J}\left(\omega_{J}\right)}{2 \pi} \exp \left[-\tilde{\beta}_{J}\left(\omega_{J}\right) H_{W}^{J}\left(Q_{J}, P_{J}\right)\right],
$$

where:

$$
\tilde{\beta}_{J}\left(\omega_{J}\right)=\frac{2 \tanh \left(\frac{\beta \omega_{J}}{2}\right)}{\omega_{J}},
$$

is a frequency-dependent inverse temperature with $\beta=1 / k_{\mathrm{B}} T$, and the Hamiltonian $H_{W}^{J}\left(Q_{J}, P_{J}\right)$ of the $J^{\text {th }}$ mode is defined in Equation (13). The thermal Wigner function of the field is:

$$
W^{T}(Q, P)=\prod_{J=1}^{\infty} W_{J}^{T}\left(Q_{J}, P_{J}\right)
$$


Equations (18)-(20) show that, in the Wigner representation, the thermal state of a free field is given in terms of an infinite product of single mode Wigner functions. Each of these single mode Wigner functions is a Boltzmann factor with a frequency-dependent temperature, as defined in Equation (19). Thus, the thermal vacuum state requires each oscillator to have a minimum average energy consistent with the temperature constraints fixed by Equation (19). In a numerical simulation, the number of oscillators in Equation (20) for the thermal state of the free field must be truncated to a finite number $N$, i.e.,

$$
\tilde{W}^{T}(Q, P)=\prod_{J=1}^{N} W_{J}^{T}\left(Q_{J}, P_{J}\right)
$$

The dynamics of this field can be simulated by first sampling the initial $\left(Q_{J}, P_{J}\right)$ from the Boltzmann factors $W_{J}^{T}\left(Q_{J}, P_{J}\right)$ for each of the $N$ modes and then propagating the modes in time by numerically solving Equation (16). Since Equation (16) generates constant energy trajectories, the thermal equilibrium distribution in Equation (21) is an invariant of the energy conserving phase space flow of the free field. It should be noted, however, that the invariance is broken when the field is coupled to another system. The lack of invariance of the thermal distribution arises ultimately from the non-conservation of the free field Hamiltonian in the presence of the coupling.

In addition to the case when the field is coupled to another system [47-57] there are a number of cases when it is desirable to simulate the dynamics of the thermal field state [27-34] on a computer. To this end, we employ a Nosé-Hoover chain (NHC) thermostat [44,45], which may be theoretically defined in terms of a quasi-Lie bracket [63-66]. In general, NHC thermostats are used to increase the ergodicity of the dynamics of non-ergodic systems, such as a system of harmonic oscillators. In NHC thermostatted dynamics, the phase space point of oscillator $J$ is extended as follows:

$$
\tilde{X}_{J}=\left(Q_{J}, \xi_{1}^{J}, \xi_{2}^{J}, \ldots, \xi_{n}^{J}, P_{J}, \chi_{1}^{J}, \chi_{2}^{J}, \ldots, \chi_{n}^{J}\right)
$$

where $\xi_{K}^{J}$ and $\chi_{K}^{J}$ denote the position and momentum, respectively, of the $K^{\text {th }}$ thermostat in the chain attached to mode $J$ and $n$ defines the length of the chain. The energy of the NHC thermostat is given by:

$$
H_{N H C}(\chi, \xi)=\sum_{J=1}^{N}\left[\left(\frac{\left(\chi_{1}^{J}\right)^{2}}{2 \mathcal{M}_{1}}+N k_{\mathrm{B}} T_{J} \xi_{1}^{J}\right)+\sum_{K=2}^{n}\left(\frac{\left(\chi_{K}^{J}\right)^{2}}{2 \mathcal{M}_{K}}+k_{\mathrm{B}} T_{J} \xi_{K}^{J}\right)\right] .
$$

The temperature control of the physical coordinates, $X$, of the field by the fictitious NHC phase space coordinates, denoted collectively by $(\chi, \xi)$, is realized by solving a set of quasi-Hamiltonian equations of motion (shown below in Equation (28)). The inertial parameters $\mathcal{M}_{K}$ (where $K=1, \ldots, n$ ) control the speed of the response of the thermostat variables to the imbalance between the instantaneous kinetic energy of each mode, $P_{J}^{2} / 2 \mu_{J}$, and the kinetic energy corresponding to its desired temperature $T_{J}, k_{B} T_{J}$, where $k_{B}$ denotes the Boltzmann constant. Finally, the Hamiltonian of the field together with the NHC thermostat is:

$$
H_{t o t}=H_{(Q, P)}+H_{N H C}(\chi, \xi) .
$$

In order to illustrate the theory, for convenience, we set the number of thermostats in the NHC to $n=2$. The quasi-Hamiltonian equations of motion are given by:

$$
\frac{d}{d t} \tilde{X}_{J}(t)=-H_{t o t} \overleftarrow{\nabla} \mathcal{B}_{\mathrm{NHC}} \vec{\nabla} \tilde{X}_{J}(t)
$$


where $\nabla=\left(\partial / \partial Q_{1}, \ldots, \partial / \partial Q_{J}, \partial / \partial \xi_{1}^{J}, \partial / \partial \xi_{2}^{J}, \partial / \partial P_{J}, \partial / \partial \chi_{1}^{J}, \partial / \partial \chi_{2}^{J}, \ldots, \partial / \partial \chi_{2}^{N}\right)$ and:

$$
\mathcal{B}_{\mathrm{NHC}}=\left[\begin{array}{cccccc}
\mathbf{0} & \mathbf{0} & \mathbf{0} & \mathbf{1} & \mathbf{0} & \mathbf{0} \\
\mathbf{0} & \mathbf{0} & \mathbf{0} & \mathbf{0} & \mathbf{1} & \mathbf{0} \\
\mathbf{0} & \mathbf{0} & \mathbf{0} & \mathbf{0} & \mathbf{0} & \mathbf{1} \\
-\mathbf{1} & \mathbf{0} & \mathbf{0} & \mathbf{0} & -\mathbf{P} & \mathbf{0} \\
\mathbf{0} & -\mathbf{1} & \mathbf{0} & \mathbf{P} & \mathbf{0} & -\chi_{1} \\
\mathbf{0} & \mathbf{0} & -\mathbf{1} & \mathbf{0} & \chi_{1} & \mathbf{0}
\end{array}\right]
$$

is the antisymmetric matrix that generalizes the symplectic matrix, defining the quasi-Hamiltonian NHC phase space flow (where each element of the matrix in Equation (26) is an $N \times N$ block diagonal matrix, e.g., $\mathbf{P}$ is an $N \times N$ block diagonal matrix containing the $P_{J}$ 's along the diagonal). The right hand side of Equation (25) defines the quasi-Lie bracket [63], which generalizes the Poisson (Lie) bracket to the case of NHC dynamics [44,45]. It should be noted that the coupling between the field and the NHC thermostat does not arise from the Hamiltonian in Equation (24), but from the the matrix $\mathcal{B}_{\mathrm{NHC}}$ in the quasi-Hamiltonian equations of motion in Equation (26). Finally, the initial thermal Wigner function in the extended space is given by:

$$
\tilde{W}^{T}(\tilde{X})=\prod_{J=1}^{N} W_{J}^{T}\left(Q_{J}, P_{J}\right) \prod_{K=1}^{2} \delta\left(\xi_{K}^{J}-\xi_{K}^{J}(0)\right) \delta\left(\chi_{K}^{J}-\chi_{K}^{J}(0)\right) .
$$

Its equation of motion reads [67]:

$$
\frac{\partial}{\partial t} \tilde{W}^{T}(\tilde{X}, t)=H_{t o t} \overleftarrow{\nabla} \mathcal{B}_{\mathrm{NHC}} \vec{\nabla} \tilde{W}^{T}(\tilde{X}, t)-\kappa \tilde{W}^{T}(\tilde{X}, t)
$$

where:

$$
\kappa=\sum_{K=1}^{6 \mathbf{N}} \frac{\partial \dot{X}_{K}}{\partial \tilde{X}_{K}}=\sum_{K=1}^{6 N} \sum_{\mathbf{J}=1}^{6 \mathrm{~N}} \frac{\partial \mathcal{B}_{\mathrm{NHC}}^{\mathrm{KJ}}}{\partial X_{K}} \frac{\partial H_{\text {tot }}}{\partial X_{J}}
$$

is the compressibility of the phase space. The emergence of the compressibility of the phase space in Equation (28) is a signature of the effects of the quasi-Hamiltonian evolution of the coordinates on the Wigner function. To understand fully how the compressibility arises, one can introduce the Wigner-Liouville operator $i \mathcal{L}=-H_{\text {tot }} \overleftarrow{\nabla} \mathcal{B}_{\mathrm{NHC}} \vec{\nabla}$ from Equation (25) into the expression for the Wigner phase space average of a generic operator $\mathcal{O}$ in the Heisenberg picture, i.e.,

$$
\langle\mathcal{O}(t)\rangle=\int d X W^{\mathrm{T}}(X) e^{i \overrightarrow{\mathcal{L}} t} \mathcal{O}_{\mathrm{W}}(X) .
$$

Upon integrating by parts, one obtains the corresponding expression in the Schrödinger picture:

$$
\langle\mathcal{O}(t)\rangle=\int d X W^{\mathrm{T}}(X) e^{-i \overleftarrow{\mathcal{L}}^{\dagger} t} \mathcal{O}_{\mathrm{W}}(X)
$$

where the adjoint Wigner-Liouville operator is given by:

$$
-i \mathcal{L}^{\dagger}=H_{\text {tot }} \overleftarrow{\nabla} \mathcal{B}_{\mathrm{NHC}} \vec{\nabla}-\kappa
$$

From a very different perspective, Equations (27) and (28) achieve a similar goal to that of thermo field dynamics: the thermal state is represented in a manner analogous to a pure state, but in a space with additional dimensions, viz., the extended Fock space in thermo field dynamics or the extended 
Wigner phase space in the current formulation. The main difference is that the additional coordinates of the NHC thermostats in the extended Wigner phase space are treated classically.

The possibility of separately controlling the temperature of each mode by means of an NHC thermostat may lead to the design of computational experiments that, to our knowledge, have not been performed before for quantum processes. For example, starting from the quantum initial condition in Equation (27) and then setting $\tilde{\beta}_{J}=\beta$ for $J=1, \ldots, N$ (which corresponds to the classical limit) in the NHC dynamics generate a nonequilibrium situation, from which the field would ultimately reach its classical thermal state. In a situation where the field is no longer isolated, it would be interesting to investigate how the time dependent classical limit of the field would modify the dynamics of the coupled system. Moreover, the classical limit may be applied to groups of modes so that one can study how each group affects the dynamics of the coupled system.

\section{Conclusions}

In this paper, we discussed how pure and thermal states of free bosonic fields [27-34] can be represented theoretically and simulated computationally. The simulation protocol, which involves an NHC thermostat $[44,45]$ coupled to each field mode, propagates the dynamics of a thermal state living in an extended Wigner space [67]. This is conceptually similar to what is done in thermo field dynamics [24-26].

For simplicity, the theory was only applied to the free field case [38], in order to demonstrate the technicalities associated with coupling a different NHC thermostat $[44,45]$ to each field mode. However, this theory is intended for situations where the field is coupled to a spin system [47-57]. In this case, the use of NHC thermostats in the dynamics of the thermal state of the field would make it possible to simulate processes that, to our knowledge, have not been investigated so far. For example, NHC dynamics could allow one to simulate the transition from a vacuum field state to a thermal vacuum state (or to thermally excited states of the field). Then, one could study changes in the spin system's transport properties in response to various field thermal processes. Such applications are left for future work.

Author Contributions: The authors contributed equally to this work.

Funding: This research received no external funding.

Conflicts of Interest: The authors declare no conflict of interest.

\section{Abbreviations}

The following abbreviations are used in this manuscript:

NHC Nosé-Hoover chain

\section{References}

1. Sen, S.; Gupta, K.S.; Coey, J.M.D. Mesoscopic structure formation in condensed matter due to vacuum fluctuations. Phys. Rev. B 2015, 92, 155115. [CrossRef]

2. Sen, S.; Gupta, K.S. Observable consequences of zero-point energy. Mod. Phys. Lett. A 2017, $32,1750217$. [CrossRef]

3. Canaguier-Durand, A.; Devaux, E.; George, J.; Pang, Y.; Hutchison, J.A.; Schwartz, T.; Genet, C.; Wilhelms, N.; Lehn, J.-M.; Ebbesen, T.W. Thermodynamics of Molecules Strongly Coupled to the Vacuum Field. Angew. Chem. Int. Ed. 2015, 52, 10533-10536. [CrossRef]

4. Shalabney, A.; George, J.; Hutchison, J.; Pupillo, G.; Genet, C.; Ebbesen, T.W. Coherent coupling of molecular resonators with a microcavity mode. Nat. Commun. 2015, 6, 5981. [CrossRef] [PubMed]

5. Hutchison, J.A.; Liscio, A.; Schwartz, T.; Canaguier-Durand, A.; Genet, C.; Palermo, V.; Samorí, P.; Ebbesen, T.W. Tuning the Work-Function Via Strong Coupling. Adv. Mater. 2013, 25, 2481-2485. [CrossRef] [PubMed] 
6. Orgiu, E.; George, J.; Hutchison, J.A.; Devaux, E.; Dayen, J.F.; Doudin, B.; Stellacci, F.; Genet, C.; Schachenmayer, J.; Genes, C.; et al. Conductivity in organic semiconductors hybridized with the vacuum field. Nat. Mater. 2013, 14, 1123. [CrossRef]

7. Huang, K.; Low, H.-B.; Tung, R.-S. Scalar field cosmology: I. Asymptotic freedom and the initial-value problem. Class. Quantum Gravity 2012, 29, 155014. [CrossRef]

8. Huang, K.; Low, H.-B.; Tung, R.-S. Scalar field cosmology II: Superfluidity, quantum turbulence, and inflation. Int. J. Mod. Phys. A 2012, 27 1250154. [CrossRef]

9. Huang, K.; Xiong, C.; Zhao, X. Scalar-field theory of dark matter. Int. J. Mod. Phys. A 2014, $29,1450074$. [CrossRef]

10. Huang, K. A Superfluid Universe; World Scientific: Singapore, 2016. [CrossRef]

11. Dirac, P.A.M. Is there an æther? Nature 1951, 168, 906-907. [CrossRef]

12. Dirac, P.A.M. Is there an æther? Nature 1952, 169, 702. [CrossRef]

13. Sinha, K.P.; Sivaram, C.; Sudarshan, E.C.G. Aether as a superfluid state of particle-antiparticle pairs. Found. Phys. 1976, 6, 65-70. [CrossRef]

14. Sinha, K.P.; Sivaram, C.; Sudarshan, E.C.G. The superfluid vacuum state, time-varying cosmological constant, and nonsingular cosmological models. Found. Phys. 1976, 6, 717-726. [CrossRef]

15. Sinha, K.P.; Sudarshan, E.C.G. The superfluid as a source of all interactions. Found. Phys. 1978, 8, 823-831. [CrossRef]

16. Eastham, P.R.; Littlewood, P.B. Bose condensation of cavity polaritons beyond the linear regime: The thermal equilibrium of a model microcavity. Phys. Rev. B 2001, 64, 235101. [CrossRef]

17. Zinn-Justin, J. Quantum Field Theory and Critical Phenomena; Oxford University Press: Oxford, UK, 1993.

18. Le Bellac, M. Quantum and Statistical Field Theory; Oxford University Press: Oxford, UK, 1992.

19. Ai, W.-Y. Correspondence between thermal and quantum vacuum transitions around horizons. J. High Energy Phys. 2019, 2019, 164. [CrossRef]

20. Canko, D.; Gialamas, I.; Jelic-Cizmek, G.; Riotto, A.; Tetradis, N. On the Catalysis of the Electroweak Vacuum Decay by Black Holes at High Temperature. Eur. Phys. J. C 2018, 78, 328. [CrossRef]

21. Gorbunov, D.; Levkov, D.; Panin, A. Fatal youth of the Universe: Black hole threat for the electroweak vacuum during preheating. J. Cosmol. Astropart. Phys. 2017, 10, 016. [CrossRef]

22. Terc, H.; Ribeiro, S.; Pezzutto, M.; Omar, Y. Quantum thermal machines driven by vacuum forces. Phys. Rev. E 2017, 95, 022135. [CrossRef]

23. Mukaida, K.; Yamada, M. False Vacuum Decay Catalyzed by Black Holes. Phys. Rev. D 2017, 96, 103514. [CrossRef]

24. Umezawa, H.; Matsumoto, H.; Tachiki, M. Thermo Field Dynamics and Condensed States; North Holland: Amsterdam, The Netherlands, 1982.

25. Umezawa, H. Advanced Field Theory: Micro, Macro, and Thermal Physics; American Institute of Physics: Woodbury, NY, USA, 1995.

26. Das, A. Finite Temperature Field Theory; World Scientific Publishing Co., Pte. Ltd.: Singapore, 1997. [CrossRef]

27. Tononi, A. Zero-Temperature Equation of State of a Two-Dimensional Bosonic Quantum Fluid with Finite-Range Interaction. Condens. Matter 2019, 4, 20. [CrossRef]

28. Chiquillo, E. Low-dimensional self-bound quantum Rabi-coupled bosonic droplets. Phys. Rev. A 2019, 99, 051601. [CrossRef]

29. Chiquillo, E. Equation of state of the one- and three-dimensional Bose-Bose gases. Phys. Rev. A 2018, 97, 063605. [CrossRef]

30. Tononi, A.; Cappellaro, A.; Salasnich, L. Condensation and superfluidity of dilute Bose gases with finite-range interaction. New J. Phys. 2018, 20, 125007. [CrossRef]

31. Cappellaro, A.; Salasnich, L. Finite-range corrections to the thermodynamics of the one-dimensional Bose gas. Phys. Rev. A 2017, 96, 063610. [CrossRef]

32. Cappellaro, A.; Salasnich, L. Thermal field theory of bosonic gases with finite-range effective interaction. Phys. Rev. A 2017, 95, 033627. [CrossRef]

33. Rivas, A.; Martin-Delgado, M.A. Topological Heat Transport and Symmetry-Protected Boson Currents. Sci. Rep. 2017, 7, 6350. [CrossRef] 
34. Wang, J.-S.; Agarwalla, B.K.; Thingna, H.L.J. Nonequilibrium Green's function method for quantum thermal transport. Front. Phys. 2014, 9, 673-697. [CrossRef]

35. Braasch, W.F., Jr.; Friedman, O.D.; Rimberg, A.J.; Miles, P.B. Wigner current for open quantum systems. Phys. Rev. A 2019, 100, 012124. [CrossRef]

36. Bialynicki-Birula, I.; Varró, S.; Ádám, P.; Biró, T.S.; Barnaföldi, G.G.; Lévai, P. Relativistic Wigner functions. EPJ Web Conf. 2014, 78, 01001. [CrossRef]

37. Bialynicki-Birula, I. Quantum fluctuations of geometry in a hot Universe. Class. Quantum Gravity 2015, 32, 215015. [CrossRef]

38. Bialynicki-Birula, I. The Wigner functional of the electromagnetic field. Opt. Commun. 2000, 179, $237-246$. [CrossRef]

39. Mrówczyński, S.; Müller, B. Wigner functional approach to quantum field dynamics. Phys. Rev. D 1994, 50, 7542-7552. [CrossRef] [PubMed]

40. de Groot, S.R. Foundations of Electrodynamics; North Holland Publishing Company: Amsterdam, The Netherlands, 1972.

41. Weinbub, J.; Ferry, D.K. Recent advances in Wigner function approaches. Appl. Phys. Rev. 2018, 5, 041104. [CrossRef]

42. Ballentine, L. Quantum Mechanics: A Modern Development; World Scientific Publishing Co., Pte. Ltd.: Singapore, 1998. [CrossRef]

43. Wigner, E. On the Quantum Correction For Thermodynamic Equilibrium. Phys. Rev. 1932, 40, 749-759. [CrossRef]

44. Martyna, G.J.; Klein, M.L.; Tuckerman, M. Nosé-Hoover chains: The canonical ensemble via continuous dynamics. J. Chem. Phys. 1992, 97, 2635-2643. [CrossRef]

45. Tobias, D.J.; Martyna, G.J.; Klein, M.L.J. Molecular Dynamics Simulations of a Protein in the Canonical Ensemble. Phys. Chem. 1993, 97, 12959-12966. [CrossRef]

46. Hillery, M.; O'Connell, R.F.; Scully, M.O.; Wigner, E.P. Distribution Functions in Physics: Fundamentals. Phys. Rep. 1984, 106, 121-167. [CrossRef]

47. von Lindenfels, D.; Gräb, O.; Schmiegelow, C.T.; Kaushal, V.; Schulz, J.; Mitchison, M.T.; Goold, J.; Schmidt-Kaler, F.; Poschinger, U.G. Spin Heat Engine Coupled to a Harmonic-Oscillator Flywheel. Phys. Rev. Lett. 2019, 123, 080602. [CrossRef]

48. Xu, X.; Choo, K.; Balachandran, V.; Poletti, D. Transport and Energetic Properties of a Ring of Interacting Spins Coupled to Heat Baths. Entropy 2019, 21, 228. [CrossRef]

49. Robert-de-Saint-Vincent, M.; Pedri, P.; BLaburthe-Tolra, B. Dissipative cooling of spin chains by a bath of dipolar particles. New J. Phys. 2018, 20, 073037. [CrossRef]

50. McCaul, G.M.G.; Lorenz, C.D.; Kantorovich, L. Driving spin-boson models from equilibrium using exact quantum dynamics. Phys. Rev. B 2018, 97, 224310. [CrossRef]

51. Lambert, N.; Ahmed, S.; Cirio, M.; Nori, F. Modelling the ultra-strongly coupled spin-boson model with unphysical modes. Nat. Commun. 2019, 10, 3721. [CrossRef] [PubMed]

52. Saryal, S.; Friedman, H.M.; Segal, D.; Agarwalla, B.K. Thermodynamic uncertainty relation in thermal transport. Phys. Rev. E 2019, 100, 042101. [CrossRef]

53. Segal, D. Current fluctuations in quantum absorption refrigerators. Phys. Rev. E 2018, 97, 052145. [CrossRef]

54. Kilgour, M.; Dvira Segal, D. Coherence and decoherence in quantum absorption refrigerators. Phys. Rev. E 2018, 98, 012117. [CrossRef]

55. Mitchison, M.T. Quantum thermal absorption machines: Refrigerators, engines and clocks. Contemp. Phys. 2019, 60, 164-187. [CrossRef]

56. Yamamoto, T.; Kato, M.; Kato, T.; Saito, K. Heat transport via a local two-state system near thermal equilibrium. New J. Phys. 2018, 20, 093014. [CrossRef]

57. Liu, J.; Hsieh, C.-Y.; Segal, D.; Hanna, G. Heat transfer statistics in mixed quantum-classical systems. J. Chem. Phys. 2018, 149, 224104. [CrossRef]

58. Goldstein, H. Classical Mechanics; Addison-Wesley: New York, NY, USA, 1980.

59. Peskin, M.E.; Schroeder, D.V. An Introduction to Quantum Field Theory; Perseus Books Publishing, L.L.C.: Reading, MA, USA, 1995.

60. Mandl, F.; Shaw, G. Quantum Field Theory; John Wiley \& Sons: New York, NY, USA, 2010.

61. Ramond, P. Field Theory: A Modern Primer; The Benjamin/Cummings Pub. Co.: Reading, MA, USA, 1981. 
62. Altland, A.; Simons, B.D. Condensed Matter Field Theory; Cambridge University Press: Cambridge, UK, 2010. [CrossRef]

63. Sergi, A.; Ferrario, M. Non-Hamiltonian equations of motion with a conserved energy. Phys. Rev. E 2001, 64, 056125.

64. Sergi, A. Non-Hamiltonian Commutators in Quantum Mechanics. Phys. Rev. E 2005, 72, 066125. [CrossRef] [PubMed]

65. Sergi, A. Deterministic constant-temperature dynamics for dissipative quantum systems. J. Phys. A Math. Theor. 2007, 40, F347-F354. [CrossRef]

66. Sergi, A.; Hanna, G.; Grimaudo, R.; Messina, A. Quasi-Lie Brackets and the Breaking of Time-Translation Symmetry for Quantum Systems Embedded in Classical Baths. Symmetry 2018, 10, 518.

67. Sergi, A.; Petruccione, F. Nosé-Hoover dynamics in quantum phase space. J. Phys. A 2008, 41, 355304. [CrossRef]

(C) 2019 by the authors. Licensee MDPI, Basel, Switzerland. This article is an open access article distributed under the terms and conditions of the Creative Commons Attribution (CC BY) license (http:/ / creativecommons.org/licenses/by/4.0/). 